\title{
Producción de Pleurotus ostreatus sobre residuos sólidos lignocelulósicos de diferente procedencia
}

\author{
Juan Pablo Garzón Gómez', Jairo Leonardo Cuervo Andrade² \\ 1. Ingeniero Agrónomo, facultad de Agronomía, Universidad Nacional de Colombia. \\ 2. Profesor asociado facultad de Agronomía, Universidad Nacional de Colombia. \\ Correspondencia: jlcuervoa@unal.edu.co. \\ Recibido: 25-09-08 / Aceptado: 20-11-08
}

\section{Resumen}

Se hizo un cultivo del hongo Pleurotus ostreatus sobre cuatro residuos sólidos de diferente procedencia usados como sustratos. Éstos fueron bagazo de caña de azúcar, tallo de maíz, aserrín y sobras de café de consumo humano. Se evaluó el efecto de los cuatro sustratos de forma individual y en mezclas sobre la producción del hongo y en mezclas sobre la producción del hongo a través de indicadores como la eficiencia biológica, el rendimiento, el número de días en periodo de incubación, el número de días para la aparición de primordios, la frecuencia y el porcentaje de peso de cada cuerpo fructífero y la productividad. El rendimiento de los sustratos que tuvieron café tanto individualmente como en las mezclas varió entre los $265 \mathrm{~g}$ a $409 \mathrm{~g}$ y fueron significativamente más altos $(p<0,05)$ que los sustratos que no lo tenían en los cuales varió entre I, $5 \mathrm{~g}$ y $154 \mathrm{~g}$.

Comparando los diferentes sustratos usados en este ensayo, se pudo observar que al mezclar el café con bagazo de caña de azúcar o con tallo de maíz se obtuvieron los mejores resultados. En estos sustratos el número de días de incubación fue entre 7 y 16 días menor y el número de días para la aparición de primordios fue entre I I y 54 días menor respecto de los demás sustratos. Se obtuvieron eficiencias biológicas que variaron entre el 4,0 y el $48 \%$, mientras que en los demás sustratos se obtuvieron eficiencias biológicas que variaron entre el 0,5 y $36 \%$. La productividad estuvo entre 0,715 y $0,905 \mathrm{~kg}$ de hongos frescos por cada $100 \mathrm{~kg}$ de sustrato seco al día, mientras que en los demás sustratos se obtuvieron productividades que variaron entre $0,324 \mathrm{~kg}$ y $0,494 \mathrm{~kg}$ de hongos frescos por cada $100 \mathrm{~kg}$ de sustrato seco al día.

Palabras clave: biorremedación, compostaje, cuerpos fructíferos, degradación, eficiencia biológica, Pleurotus spp.

\section{Abstract \\ Production of Pleurotus ostreatus on solid lignocellulosic wastes from different source \\ A culture of the fungus Pleurotus ostreatus was grown on four solid wastes (sugar cane bagasse, maize stem, sawdust, and coffee waste of human consumption) and was used as substrate. The effects of using these four wastes were evaluated individually and in mixtures on the production of the fungus through indicators such as biological efficiency, yield, incubation period, number of days for the appearance of primordium, frequency and percentage of weight of each fruiting body, and productivity. The yield of coffee substrates that, both individually}


and in mixtures, varied between 265g to 409g and was significantly higher $(p<0.05)$ than the substrates that did not have it, in which it varied between $1.5 \mathrm{~g}$ and $154 \mathrm{~g}$

Comparing the different substrates used in this test, it was observed that the best results were obtained when mixing coffee with sugar cane bagasse or maize stem. In these substrates the number of days of incubation was between 7 and 16 fewer days and the number of days for the appearance of primordium was between II and 54 fewer days with respect to the other substrates. Biological efficiencies were obtained that varied between the 4.0 and $48 \%$, whereas in the other substrates biological efficiencies were obtained that varied between 0.5 and $36 \%$. The productivity was between 0.715 and $0.905 \mathrm{~kg}$ of fresh fungi per $100 \mathrm{~kg}$ of dry substrate to the day, while in the other substrates productivities were obtained that varied between $0.324 \mathrm{~kg}$ and $0.494 \mathrm{~kg}$ of fresh fungi per $100 \mathrm{~kg}$ of dry substrate to the day.

Key words: biological efficiency, compost, degradation, fruiting bodies, Pleurotus spp.

\section{Introducción}

El cultivo de hongos comestibles del género Pleurotus spp., comúnmente conocidos como hongos ostra u orellanas, fue realizado por primera vez en el mundo a principios del siglo pasado y se ha incrementado en las ultimas cinco décadas, alcanzando el 14,2\% de la producción total de hongos comestibles en el mundo en el año de 1997, siendo China el principal productor con el $86,8 \%$ de la producción mundial y con cerca de 800.000 toneladas producidas al año (1).

El cultivo de $P$. ostreatus fue iniciado en Colombia hacia 1990 en el Laboratorio de Microbiología de la Universidad de Antioquia por el microbiólogo Fabio Pineda, con la asesoría del micólogo Gastón Guzmán (2). Debido a la gran cantidad de residuos lignocelulósicos que se generan en el cultivo e industrialización del café, diversos estudios acerca del cultivo de hongos del género Pleurotus spp., se han realizado en la zona cafetera de Colombia desde la década de los noventa (3).

El micelio de este hongo puede crecer en una temperatura entre 0 y $35^{\circ} \mathrm{C}$, con temperatura óptima de $30{ }^{\circ} \mathrm{C}$, y en un rango de $\mathrm{pH}$ entre 5,5 y 6,5 y se ha observado que después de cosechar los cuerpos fructíferos de $P$. ostreatus, en los materiales usados como sustratos las cantidades finales de hemicelulosa, celulosa y lignina se han reducido en un $80 \%$ sugiriendo que todos los materiales que contienen estos compuestos, pobres en nitrógeno pueden ser usados como sustratos para Pleurotus spp. (4). Los hongos del género Pleurotus spp. pueden crecer con relaciones $\mathrm{C} / \mathrm{N}$ entre 30 y 300 (5). La relación $\mathrm{C} / \mathrm{N}$ óptima del sustrato depende de la fase en la que se encuentra el hongo, altas relaciones $\mathrm{C} / \mathrm{N}$ favorecen el crecimiento micelial y bajas relaciones favorecen el desarrollo de cuerpos fructíferos (6).

El contenido de humedad en el sustrato para el desarrollo de los hongos debe estar entre el 50 y el 80\%, la fructificación suele darse en condiciones normales cuando se tiene un $20 \%$ de oxígeno y una concentración de $\mathrm{CO}_{2}$ no mayor de $800 \mathrm{ppm}$ en el ambiente que circunda al hongo y la humedad relativa óptima para la fructificación de P. ostreatus es de 85 a 90\% (5). Si bien la fase de incubación del hongo debe ser en la oscuridad, se ha demostrado que la eficiencia biológica (relación entre peso fresco de los hongos y peso seco del sustrato utilizado) y el rendimiento de $P$. ostreatus cultivado sobre residuos de cacao disminuyen entre un 68\% y $63 \%$ respectivamente, cuando el hongo en su fase de fructificación está expuesto a la luz en tiempos menores de 12 horas (7).

El cultivo de los hongos del género Pleurotus spp., tiene un gran atractivo debido principalmente a:

- Producen proteínas de alta calidad sobre un sustrato que consiste en materiales de desecho de carácter lignocelulosico, materiales producidos en gran cantidad en la actividad agrícola. A pesar de que la calidad de las proteínas de los hongos no es tan alta como la proteína animal, se considera que la producción de ésta es más eficiente en términos de costos, espacio y tiempo. 
- El sustrato que queda después de la cosecha del hongo llamado compost agotado, puede ser usado como sustrato para hongos de otros géneros, como forraje para ganado, como acondicionador del suelo o fertilizante y en biorremediación.

- Presencia de componentes medicinales en los hongos (8)

En cuanto a características alimenticias de los hongos del género Pleurotus spp. se destaca su sabor, altas cantidades de proteína, fibra dietética, carbohidratos, minerales (fósforo, hierro, calcio), vitaminas (riboflavina, tiamina, ácido ascórbico y niacina), ácido linoleico, así como bajas concentraciones de grasas (9). El contenido de proteína cruda (\% en peso seco) de $P$. ostreatus oscila entre 10,5 a $30,4 \%$ (10), y la calidad biológica de estas proteínas en términos de contribución nutricional para la dieta humana es alta (11).

En cuanto al potencial como forraje que pueda tener un sustrato usado en cultivos de hongos del género Pleurotus spp., se ha encontrado que el mejoramiento de la digestibilidad depende de la especie del hongo, del sustrato utilizado y de las condiciones del cultivo $(12,13)$. En cuanto al uso como acondicionador del suelo o fertilizante de sustratos usados en el cultivo de hongos; se ha encontrado que en el cultivo de limoncillo Cymbopogon citratus altos valores de rendimiento cuando se sembró en sustratos usados en el cultivo del hongo Pleurotus sajor-caju (14).

En cuanto a la capacidad biorremediadora de hongos del género Pleurotus spp., se ha reportado la habilidad de cepas de Pleurotus pulmonarius para biotransformar herbicidas como la atrazina e insecticidas como el endosulfan $(5,15)$. Con respecto a los efectos benéficos medicinales de $P$. ostreatus es conocida su actividad anticancerígena, efectos inmunomodulatorios, antivirales, antibióticos, antiinflamatorios y disminución en los niveles de colesterol (16). A pesar de todo lo anterior, en muchos casos estos residuos son quemados o dispuestos en rellenos sanitarios; donde los biopolímeros de lenta degradación como la celulosa permanecen durante ańos casi sin alteraciones (17).

La Universidad Nacional de Colombia, sede Bogotá, con el fin de reducir el volumen de residuos sólidos generados en su propio espacio, ha venido haciendo investigación en este campo. En un estudio en el que usaron como sustrato residuos sólidos orgánicos biodegradables producidos en el campus universitario, se encontró que el aserrín, de establos de animales, presentó un bajo grado de degradación y transformación de estos compuestos orgánicos, debido a la presencia de moléculas y compuestos como la lignina y la celulosa, que son de difícil degradación. Lo anterior sugiere que este tipo de residuos generan formas menos estables en ambientes edáficos y por lo tanto fueron calificados como menos aptos para la fertilización de cultivos cuando se les compara con otros residuos de más fácil degradación. Así mismo, se ha observado que el residuo de café de consumo humano, generado en las cafeterías, presenta una muy baja o nula degradación en el proceso de compostaje (18).

En el presente trabajo se evaluó el efecto generado por el uso de residuos de carácter lignocelulósico y de baja degradación en el proceso de compostaje generados en el Campus universitario, como lo son el aserrín de establos de animales y residuos de café de consumo humano, sobre variables de rendimiento de un cultivo de $P$. ostreatus, y así buscar otra alternativa en el manejo de este tipo de residuos. También se evaluaron residuos como el tallo de maíz producido en cultivos de la universidad y el bagazo de la caña de azúcar.

\section{Materiales y métodos}

El presente trabajo se llevó a cabo en la Facultad de Agronomía de la Universidad Nacional de Colombia, sede Bogotá, en el primer semestre del año 2007. Para el establecimiento del cultivo se construyó sobre el suelo un invernadero de $8 \mathrm{mX} 3 \mathrm{mX} 2,5 \mathrm{~m}$ de altura, de estructura metálica y con plástico negro; el cual fue dividido en dos secciones: la primera correspondió a una cuarta parte del invernadero totalmente oscura para la fase de incubación o estado vegetativo (invasión del micelio sobre el sustrato) y la segunda a las tres cuartas partes restantes del invernadero con una ventana que permitía ventilación y 12 horas de luz indirecta en el día para la fase de fructificación o estado generativo (producción de cuerpos fructíferos). En este trabajo el cultivo del hongo consistió en: preparación de los sustratos, inoculación, crecimiento del hongo y cosecha.

\section{Preparación del sustrato e inoculación}

Los residuos que se evaluaron como sustratos para el cultivo se recolectaron en la Universidad Nacional de Colombia, sede Bogotá, a excepción del bagazo de caña de 
Tabla 1. Proporciones de los residuos usados como sustratos (tratamientos) y cantidad de semilla utilizada con base al $5 \%$ del peso seco total del sustrato.

\begin{tabular}{|c|c|c|c|c|c|}
\hline \multirow[b]{2}{*}{ Tratamiento } & \multirow[b]{2}{*}{ Sustrato } & \multirow[b]{2}{*}{$\%$ en volumen } & \multicolumn{3}{|c|}{ Peso seco total } \\
\hline & & & $\%$ en peso & (g) & Semilla (5\%) \\
\hline 1 & $\mathrm{C}$ & $(100)$ & $(100)$ & 1360 & 68 \\
\hline 2 & A & $(100)$ & $(100)$ & 776 & 38,8 \\
\hline 3 & $S$ & $(100)$ & $(100)$ & 280 & 14 \\
\hline 4 & M & $(100)$ & $(100)$ & 280 & 14 \\
\hline 5 & $C+A$ & $(75+25)$ & $(84+16)$ & 1214 & 60,7 \\
\hline 6 & $C+A$ & $(50+50)$ & $(64+36)$ & 1068 & 53,4 \\
\hline 7 & $C+A$ & $\begin{array}{l}(25+75) \\
(25+25+25+\end{array}$ & $\begin{array}{l}(37+63) \\
(50+30+10+\end{array}$ & 922 & 46,1 \\
\hline 8 & $C+A+M+S$ & 25) & 10) & 674 & 33,7 \\
\hline 9 & $C+M+S$ & $(50+25+25)$ & $(82+9+9)$ & 820 & 41 \\
\hline 10 & $A+M+S$ & $(50+25+25)$ & $(74+13+13)$ & 528 & 26,4 \\
\hline 11 & $C+A+M$ & $(25+25+50)$ & $(50+29+21)$ & 674 & 33,7 \\
\hline 12 & $C+A+S$ & $(25+25+50)$ & $(50+29+21)$ & 674 & 33,7 \\
\hline
\end{tabular}

El volumen dado es el porcentaje del volumen de cada residuo de un volumen total de 5 litros.

C: Café A: Aserrín S: Bagazo de caña de azúcar M: Tallo de maíz

azúcar y fueron: café de consumo humano originado de las sobras provenientes de las cafeterías de la Universidad; aserrín originado de la renovación de establos de animales (residuo pecuario) suministrados por la Facultad de Medicina Veterinaria y Zootecnia; tallo de maíz originada de un cultivo (residuo agrícola) de la Facultad de Agronomía y bagazo originado en el procesamiento de la caña de azúcar (residuo agroindustrial) del municipio de Villeta, Cundinamarca.

El tallo de maíz y el bagazo de la caña de azúcar estuvieron almacenados 5 y 3 meses respectivamente después de la cosecha y se picaron para conseguir un tamaño de partículas de 6 a $8 \mathrm{~cm}$. Los materiales usados presentan diferentes densidades siendo mayores las del café de consumo humano y el aserrín respecto de las del tallo de maíz y el bagazo de la caña de azúcar ya que estas ultimas por unidad de masa ocupan un mayor volumen; por esta razón y con base en observaciones realizadas en ensayos preliminares se decidió elaborar todos los sustratos de tal manera que en las bolsas contenedoras ocuparan un volumen de 5L, independiente de si estuvieran compuestos de un solo residuo o de mezclas.

Cada uno de los sustratos fue pesado individualmente para posteriormente ser colocados en un recipiente con 30L de agua y realizar la mezcla durante 5 minutos, de tal manera que cada proporción en el sustrato estuviera bien mezclada y saturado de agua; después de mezclado se midió el pH y se ajustó dentro de un rango de 6 a 7 con carbonato de calcio. La mezcla fue sacada del recipiente con agua y puesta sobre una superficie durante 12 horas para que se drenara el exceso de agua.

Luego cada sustrato fue empacado en una bolsa plástica blanca de $40 \times 30 \mathrm{~cm}$, para posteriormente ser esterilizados en un autoclave a $120{ }^{\circ} \mathrm{C}$ y $15 \mathrm{lbs} / \mathrm{cm}^{2}$ durante 1 hora. Para el proceso de inoculación se utilizó semilla comercial de P. ostreatus (micelio del hongo 
sobre semilla de trigo) procedente de Bogotá y se calculó la cantidad de semilla con base al $5 \%$ del peso seco total de cada sustrato, Tabla 1. Luego de que los sustratos fueron esterilizados y que estuvieran a temperatura ambiente, se hizo la mezcla de la semilla con el sustrato dentro las bolsas y en condiciones asépticas para evitar contaminación, después de realizar la mezcla, las bolsas fueron cerradas con un nudo en la parte superior. La composición de los sustratos en los tratamientos planteados en este trabajo se dan en porcentajes del volumen de cada residuo con respecto al volumen total del sustrato por bolsa ( 51 para todos los casos) y en los porcentajes del peso de cada residuo con respecto al peso seco total del sustrato por bolsa, Tabla 1. A pesar de que el dato más importante para los cálculos es el peso seco total, los tratamientos en las tablas y figuras se van a presentar con base a su porcentaje en volumen para hacer más fácil la visualización de las proporciones de cada residuo en los sustratos.
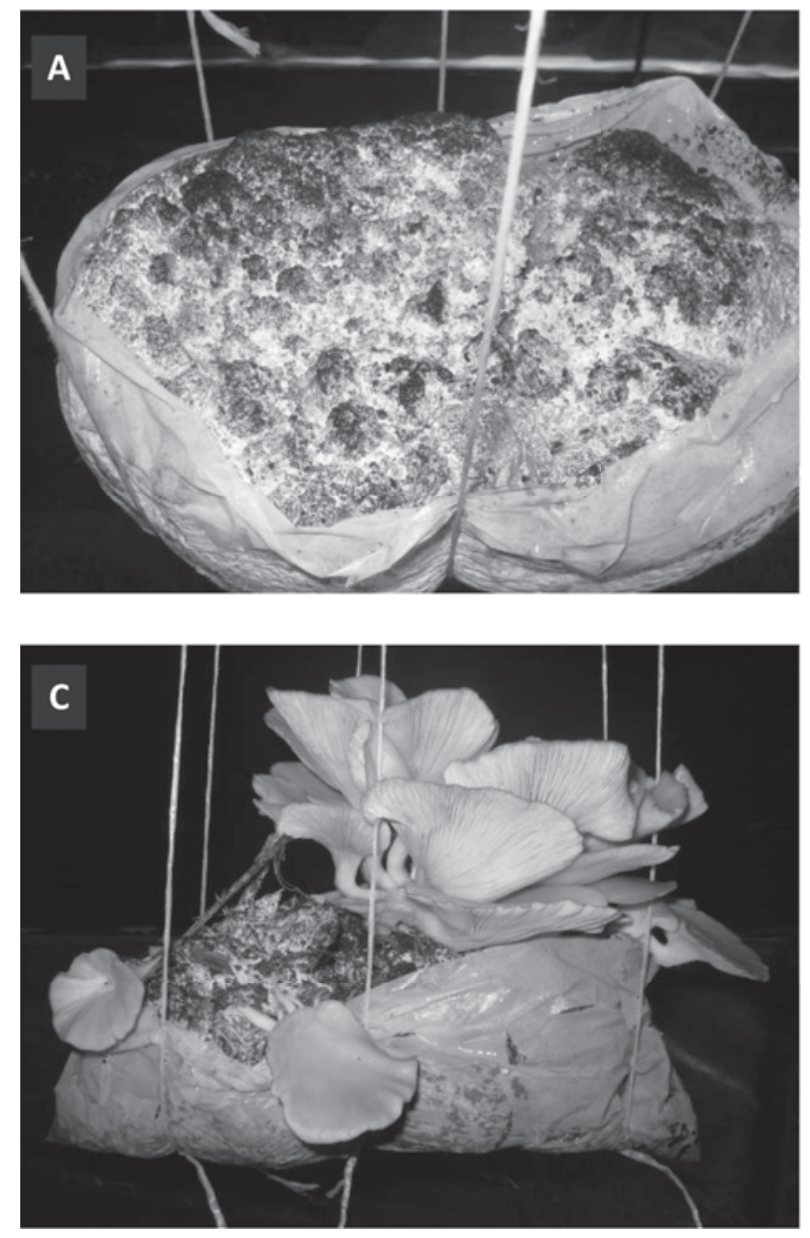

Figura 1. Producción de P. ostreatus. A: Invasión micelial (periodo de incubación) en sustrato de residuo de café solo (tratamiento 1); B: Aparición de primordios en sustrato de café mezclado con bagazo y tallo de maíz (Tratamiento 9); C y D: Cuerpos fructíferos en sustrato de café con aserrín, bagazo de caña de azúcar y tallo de maíz (tratamiento 8).

\section{Crecimiento de los cuerpos fructíferos y cosecha}

\section{Fase de incubación}

Posterior a la preparación de los sustratos, esterilización e inoculación, las bolsas fueron llevadas a un estante previamente desinfectado que se encontraba en la zona de incubación del invernadero que se construyó para este estudio, en donde se observaron temperaturas entre 7 y $34^{\circ} \mathrm{C}$ y humedad relativa entre 31 y 100\%; las bolsas permanecieron allí ocho días sin ser abiertas o movidas. Después de los ocho días, las bolsas fueron monitoreadas diariamente abriendo el nudo de la parte superior, y así mismo permitiendo aireación de los sustratos; éstas fueron volteadas diariamente para mayor homogeneidad en la humedad y aireación. Esta fase se dio por terminada cuando en los monitoreos se observó que el micelio había colonizado toda la superficie del sustrato, es decir, cuando el sustrato se veía totalmente cubierto de una masa blanquecina y algodonosa, Figura 1. En esta fase se midió el efecto de los tratamientos sobre: número de días en fase de incubación,
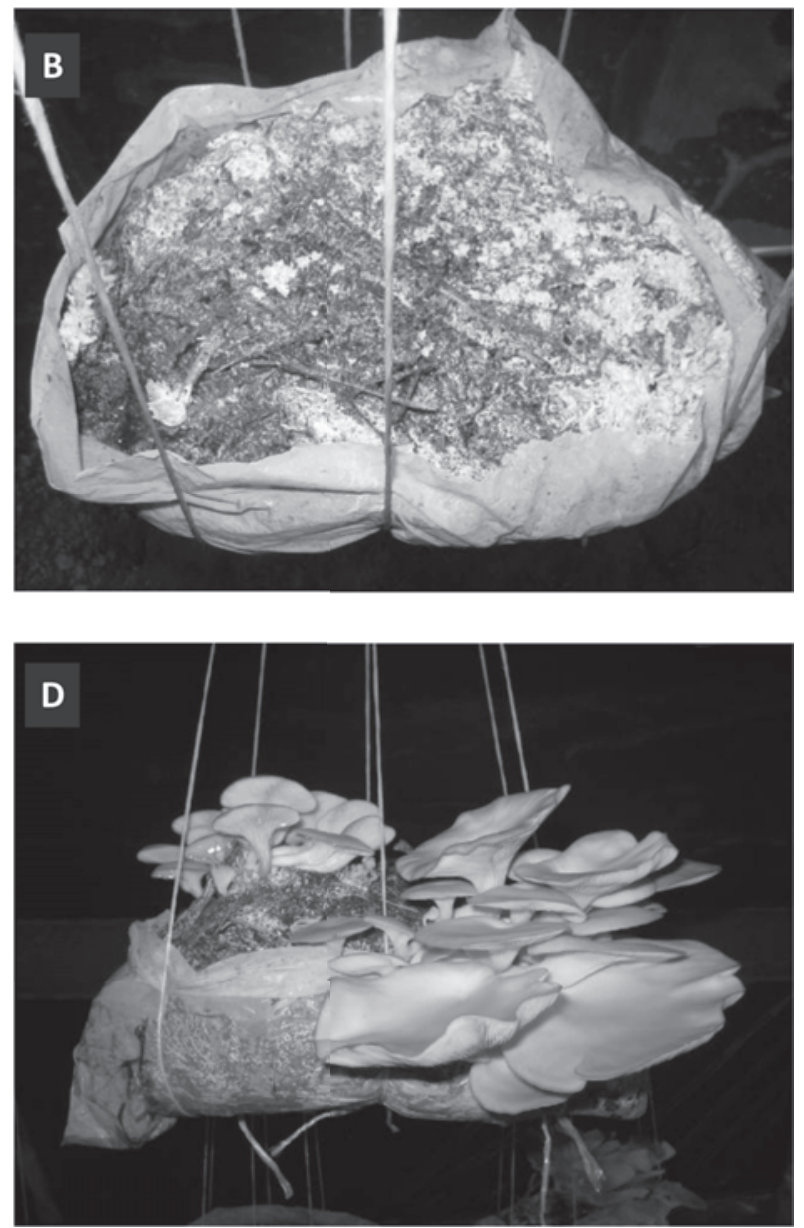
Tabla 2. Número de días en periodo de incubación de P. ostreatus para 12 tratamientos.

\begin{tabular}{cccccc}
\hline Tratami ento & Sustrato & \% en volumen & \multicolumn{3}{c}{ Días en incubación } \\
\hline 1 & C & $(100)$ & $33^{\mathrm{a}}$ & $(5,4)^{*}$ & $\mathrm{a}$ \\
2 & $\mathrm{~A}$ & $(100)$ & 42 & $(0,0)$ & $\mathrm{b}$ \\
3 & $\mathrm{~S}$ & $(100)$ & 36 & $(0,0)$ & $\mathrm{a}$ \\
4 & $\mathrm{M}$ & $(100)$ & 36 & $(0,0)$ & $\mathrm{a}$ \\
5 & $\mathrm{C}+\mathrm{A}$ & $(75+25)$ & 33 & $(3,5)$ & $\mathrm{a}$ \\
6 & $\mathrm{C}+\mathrm{A}$ & $(50+50)$ & 36 & $(0,0)$ & $\mathrm{a}$ \\
7 & $\mathrm{C}+\mathrm{A}$ & $(25+75)$ & 42 & $(0,0)$ & $\mathrm{b}$ \\
8 & $\mathrm{C}+\mathrm{A}+\mathrm{M}+\mathrm{S}$ & $(25+25+25+25)$ & 26 & $(0,0)$ & $\mathrm{C}$ \\
9 & $\mathrm{C}+\mathrm{M}+\mathrm{S}$ & $(50+25+25)$ & 31 & $(5,8)$ & $\mathrm{a}, \mathrm{C}$ \\
10 & $\mathrm{~A}+\mathrm{M}+\mathrm{S}$ & $(50+25+25)$ & 26 & $(0,0)$ & $\mathrm{C}$ \\
11 & $\mathrm{C}+\mathrm{A}+\mathrm{M}$ & $(25+25+50)$ & 26 & $(0,0)$ & $\mathrm{C}$ \\
12 & $\mathrm{C}+\mathrm{A}+\mathrm{S}$ & $(25+25+50)$ & 26 & $(0,0)$ & $\mathrm{C}$ \\
\hline
\end{tabular}

a Medias. *(Desviación estándar).

(a-c) Medias con diferente letra dentro de una misma columna son significativamente diferentes $(P<0,05)$.

C: café A: aserrín S: bagazo de caña de azúcar M: tallo de maíz.

Tabla 2 y Figuras 1,2, que es el número de días entre la inoculación y el final de la fase de incubación.

\section{Fase de fructificación}

Se observó que el micelio había colonizado toda la superficie del sustrato, entonces, las bolsas fueron llevadas a la zona de fructificación del invernadero, en donde se observaron temperaturas entre 7 y $31,5^{\circ} \mathrm{C}$ y humedad relativa entre 33 y $100 \%$, donde se procedió a colgar las bolsas de tal manera que quedaran a la mitad del total de la altura del invernadero $(1,25 \mathrm{~m})$ y que la longitud más larga del sustrato en la bolsa $(40 \mathrm{~cm})$ quedara orientada de manera horizontal, Figura 1.

Con un cortador se quitó toda la parte superior de la bolsa (en donde tenía el nudo) y se hicieron cortes verticales y horizontales en todas las superficies para aumentar aireación y promover mayor fructificación. Los sustratos fueron regados diariamente con agua potable y con un atomizador portátil para permitir una mejor distribución del agua sobre el sustrato. Las cosechas se hicieron manualmente desprendiendo los cuerpos fructíferos del sustrato cuando estos dejaban de crecer en diámetro.

En esta fase se midió el efecto de los tratamientos sobre:

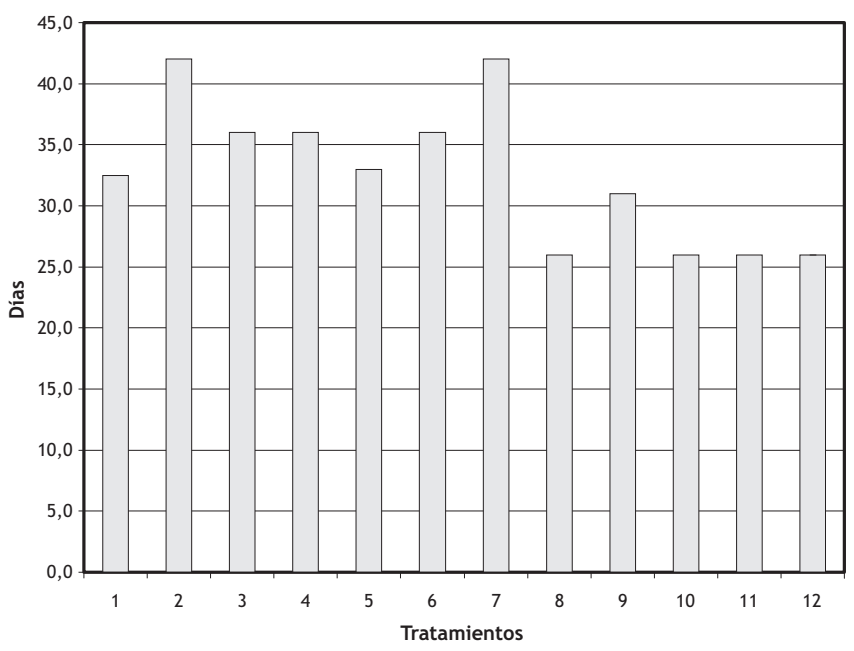

Figura 2. Número de días en periodo de incubación de P. ostreatus para 12 tratamientos.

- Número de días en aparecer los primordios de los cuerpos fructíferos para dos cosechas, Tabla 3 y Figuras 1 y 3 , que es el número de días entre la inoculación y la aparición de los primordios que formarían posteriormente los cuerpos fructíferos del hongo.

- Rendimiento de los sustratos, entendido este como el peso total de los cuerpos fructíferos para cada una y la suma de dos cosechas, Tabla 4 y Figura 4.

- Frecuencia y porcentaje del peso de cada cuerpo fructífero, Tablas 5 y 6 . 
Tabla 3. Aparición de primordios de P. ostreatus para 12 tratamientos (cosechas 1 y 2).

\begin{tabular}{|c|c|c|c|c|}
\hline \multirow{2}{*}{ Tratamiento } & \multirow[b]{2}{*}{ Sustrato } & \multirow[b]{2}{*}{$\%$ en volumen } & \multicolumn{2}{|c|}{ Aparición de primordios (días) } \\
\hline & & & Cosecha 1 & Cosecha 2 \\
\hline 1 & C & $(100)$ & $45^{\mathrm{a}}(4,8)^{*} \mathrm{a}, \mathrm{d}$ & $64^{a}(2,6)^{*} a, d, e$ \\
\hline 2 & $A$ & $(100)$ & $87 \quad(3,5) b$ & $0 \quad(0,0) B$ \\
\hline 3 & $\mathrm{~S}$ & (100) & $39(1,3) c, d$ & $58(2,4) c, d, h$ \\
\hline 4 & M & $(100)$ & $44(0,0) a, d$ & $0 \quad(0,0) B$ \\
\hline 5 & $C+A$ & $(75+25)$ & $44(2,6) a, d$ & $62(2,2) a, c, e$ \\
\hline 6 & $C+A$ & $(50+50)$ & $48 \quad(1,9) a$ & $68 \quad(2,3) A$ \\
\hline 7 & $C+A$ & $(25+75)$ & $50 \quad(2,4) a$ & $69(1,0) A$ \\
\hline 8 & $C+A+M+S$ & $(25+25+25+25)$ & $31(1,2) c$ & $48 \quad(2,4) F$ \\
\hline 9 & $C+M+S$ & $(50+25+25)$ & $34(4,5) c$ & $55(4,1) \mathrm{g}, \mathrm{h}$ \\
\hline 0 & $A+M+S$ & $(50+25+25)$ & $48 \quad(6,1) a$ & $59(4,1) c, e, h$ \\
\hline 11 & $C+A+M$ & $(25+25+50)$ & $32(2,1) c$ & $49(3,8) f, g$ \\
\hline 12 & $C+A+S$ & $(25+25+50)$ & $34(2,1) c$ & $51(4,1) f, g$ \\
\hline
\end{tabular}

a Medias. *(Desviación estándar).

(a-h) Medias con diferente letra dentro de una misma columna son significativamente diferentes $(P<0,05)$.

C: café A: aserrín S: bagazo de caña de azúcar M: tallo de maíz.

Tabla 4. Rendimiento (g) de P. ostreatus para 12 tratamientos (cosechas 1, 2 y total).

\begin{tabular}{|c|c|c|c|c|c|}
\hline \multirow[b]{2}{*}{ Tratamiento } & \multirow[b]{2}{*}{ Sustrato } & \multirow[b]{2}{*}{$\%$ en volumen } & \multicolumn{3}{|c|}{ Rendimiento (g) } \\
\hline & & & Cosecha 1 & Cosecha 2 & Total \\
\hline 1 & $\mathrm{C}$ & $(100)$ & $217,4^{a}(23,5)^{*} a, d$ & $159,8^{\mathrm{a}}(13,8)^{*} \mathrm{a}$ & $377,1^{a}(37,0)^{*} a, c$ \\
\hline 2 & $A$ & $(100)$ & $37,6(43,4) b, c$ & $0,0 \quad(0,0) b$ & $37,6(43,4) b$ \\
\hline 3 & $S$ & $(100)$ & $47,1(13,8) b, c$ & $11,1 \quad(7,8) b$ & $58,2(10,2) b$ \\
\hline 4 & M & $(100)$ & $1,5 \quad(3,0) c$ & $0,0 \quad(0,0) b$ & $1,5 \quad(3,0) b$ \\
\hline 5 & $C+A$ & $(75+25)$ & $245,8 \quad(40,9) a, d$ & $163,3(23,3) a$ & $409,0(18,7) \mathrm{c}$ \\
\hline 6 & $C+A$ & $(50+50)$ & $262,8(44,7) d$ & $119,8(20,6) a, c$ & $382,6(41,1) a, c$ \\
\hline 7 & $C+A$ & $(25+75)$ & $207,4(27,1) a, d$ & $87,8 \quad(9,8) c, d$ & $295,2(31,0) d, e$ \\
\hline 8 & $C+A+M+S$ & $(25+25+25+25)$ & $229,5(20,7) a, d$ & $97,0(26,4) c, e, g$ & $326,5(23,2) a, d, e$ \\
\hline 9 & $C+M+S$ & $(50+25+25)$ & $229,3(41,3) a, d$ & $139,5(30,6) a, e, f$ & $368,8 \quad(39,7) a, c, d$ \\
\hline 10 & $A+M+S$ & $(50+25+25)$ & $86,1(16,0) b$ & $67,9(15,7) d, g, h$ & $153,9(28,8) f$ \\
\hline 11 & $C+A+M$ & $(25+25+50)$ & $172,1 \quad(28,7) a$ & $92,7(25,2) c, h$ & $264,8(49,1) \mathrm{e}$ \\
\hline 12 & $C+A+S$ & $(25+25+50)$ & $180,6(28,5) a$ & $94,7(15,0) c, f, h$ & $275,3(31,0) \mathrm{e}$ \\
\hline
\end{tabular}

a Medias. *(Desviación estándar).

(a-h) Medias con diferente letra dentro de una misma columna son significativamente diferentes $(P<0,05)$

C: café A: aserrín S: bagazo de caña de azúcar M: tallo de maíz. 


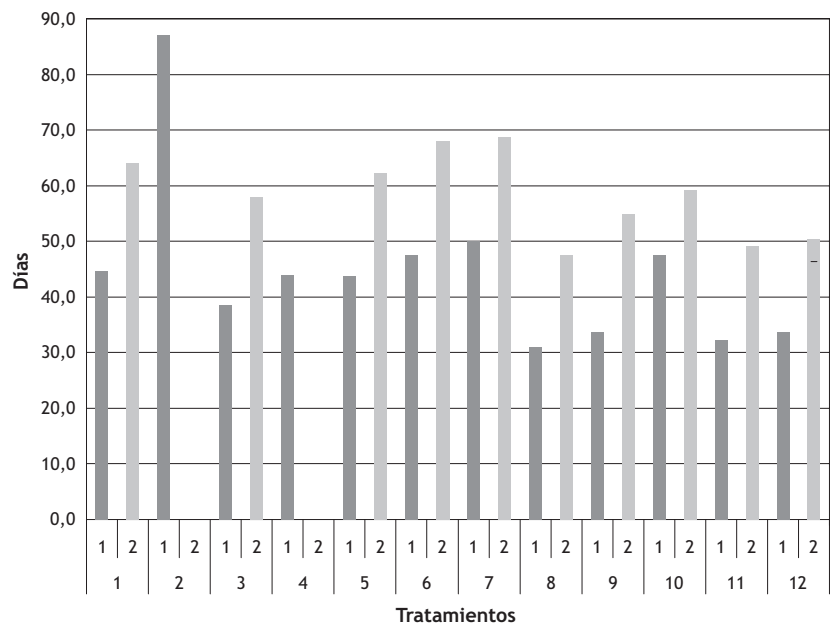

Figura 3. Aparición de primordios de P. ostreatus para 12 tratamientos (cosechas 1 y 2 ).

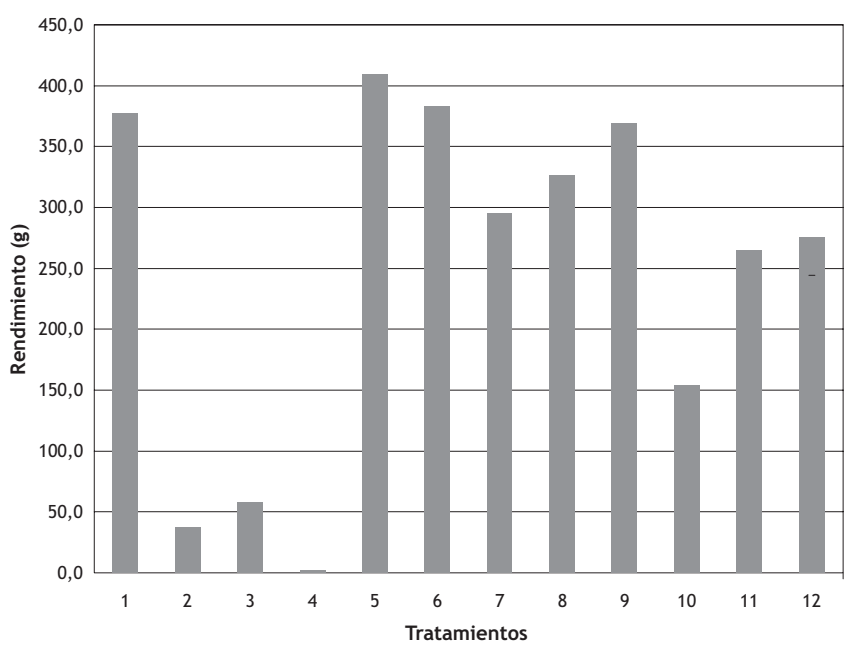

Figura 4. Rendimiento total (g) de P. ostreatus para 12 tratamientos (Total de 2 cosechas).

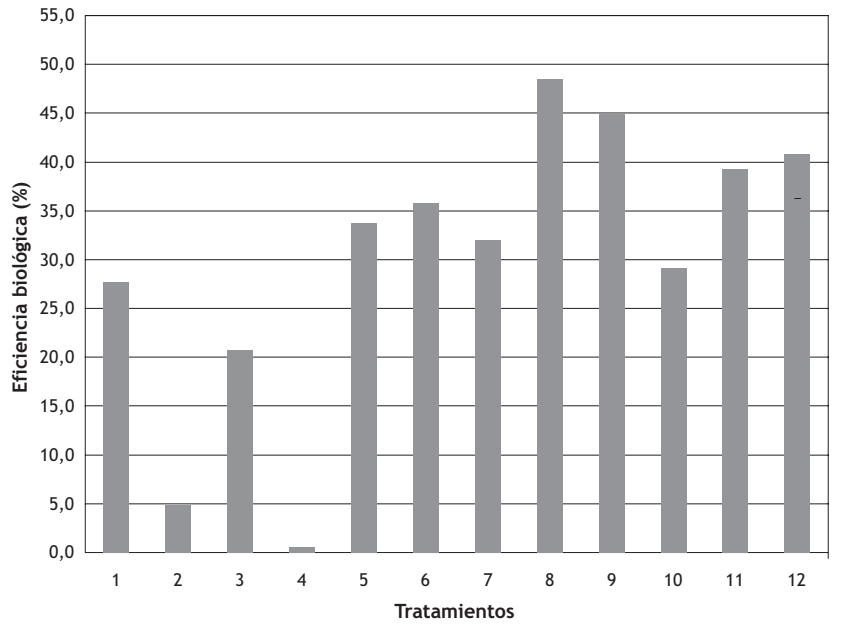

Figura 5. Eficiencia biológica (\%) de P. ostreatus para 12 tratamientos (Total de 2 cosechas).
- Eficiencia biológica, entendida esta como la relación entre el peso fresco de los cuerpos fructíferos y el peso seco del sustrato usado para su producción, para cada una y la suma de dos cosechas, Tabla 7 y Figura 5. $\mathrm{EB}=($ Peso fresco de los hongos $(\mathrm{g}) /$ Peso seco del sustrato (g)) X 100

- Productividad, entendida esta como los kilogramos de cuerpos fructíferos frescos producidos por cada 100 kilogramos de sustrato seco en el día, Tabla 8.

Productividad $=(\mathrm{kg}$ cuerpos fructíferos $/ 100 \mathrm{~kg}$ sustrato seco por día)

Todas las variables se evaluaron sobre cuatro muestras por tratamiento.

\section{Análisis estadístico}

Para determinar diferencias significativas entre los tratamientos, los datos fueron procesados y analizados con los programas Microsoft Excel y SAS, y para las variables que mostraron diferencias estadísticamente significativas se les hizo comparaciones múltiples con el procedimiento de Tukey a un nivel $\mathrm{p}=0,05$.

\section{Resultados y discusión}

\section{Número de días en incubación}

Los resultados de este parámetro se pueden observar en la Tabla 2 y Figura 2. Se puede apreciar que en los sustratos donde más rápidamente se completó la invasión del micelio, fueron las mezclas de café con o sin aserrín junto con el bagazo de la caña de azúcar y/o el tallo de maíz (tratamientos 8, 9, 10,11 y 12) con finalización del periodo de incubación a los 26 días después de la inoculación, los cuales presentaron diferencias significativas $(\mathrm{P}<0,05)$ con los demás tratamientos, a excepción del tratamiento 9 que no tuvo diferencias estadísticamente significativas con los tratamientos 1,5 y 6 y los cuales presentan una alta proporción de café respecto de los tratamientos 8, 10, 11 y 12; éste tratamiento tampoco presentó diferencias estadísticamente significativas respecto de los tratamientos 3 y 4 .

También se puede apreciar que los sustratos donde más se demoró la invasión micelial con diferencias estadísticamente significativas respecto de los demás, fueron los tratamientos 2 y 7 que tuvieron el aserrín en alta proporción pero sin bagazo de caña de azúcar o tallo 
Tabla 5. Clasificación de los cuerpos fructíferos por peso (g) de P. ostreatus para 12 tratamientos.

\begin{tabular}{cccccccccccccccc}
\hline Grado & $\mathbf{1}$ & $\mathbf{2}$ & $\mathbf{3}$ & $\mathbf{4}$ & $\mathbf{5}$ & $\mathbf{6}$ & $\mathbf{7}$ & $\mathbf{8}$ & $\mathbf{9}$ & $\mathbf{1 0}$ & $\mathbf{1 1}$ & $\mathbf{1 2}$ & $\mathbf{1 3}$ & $\mathbf{1 4}$ & $\mathbf{1 5}$ \\
\hline Peso & $1 \mathrm{a}$ & $6 \mathrm{a}$ & $11 \mathrm{a}$ & $16 \mathrm{a}$ & $21 \mathrm{a}$ & $26 \mathrm{a}$ & $31 \mathrm{a}$ & $36 \mathrm{a}$ & $41 \mathrm{a}$ & $46 \mathrm{a}$ & $51 \mathrm{a}$ & $56 \mathrm{a}$ & $61 \mathrm{a}$ & $66 \mathrm{a}$ & $71 \mathrm{a}$ \\
(g) & 5 & 10 & 15 & 20 & 25 & 30 & 35 & 40 & 45 & 50 & 55 & 60 & 65 & 70 & 75 \\
\hline
\end{tabular}

Tabla 6. Frecuencia y porcentaje de los cuerpos fructíferos de P. ostreatus para 12 tratamientos (total de 2 cosechas).

\begin{tabular}{|c|c|c|c|c|c|c|c|c|c|c|c|c|c|c|c|}
\hline \multicolumn{15}{|c|}{ Grado } & \multirow[b]{2}{*}{ Total } \\
\hline & Tratamiento & 1 & 2 & 3 & 4 & 5 & 6 & 7 & 8 & 9 & 11 & 12 & 13 & 15 & \\
\hline $\mathrm{F}$ & & 67 & 41 & 19 & 11 & 14 & 3 & & 1 & 3 & & & & & 159 \\
\hline$P$ & 1 & 42 & 25,8 & 11,95 & $6,918 \varepsilon$ & 8,805 & 1,887 & & 0,6291 & 1,887 & & & & & 100 \\
\hline $\mathrm{F}$ & & 2 & 4 & 2 & 1 & 1 & 2 & & & & & & & & 12 \\
\hline$P$ & 2 & 17 & 33,3 & 16,67 & 8,333 \& & 8,333 & 16,67 & & & & & & & & 100 \\
\hline $\mathrm{F}$ & & 44 & 10 & 3 & 1 & & & & & & & & & & 58 \\
\hline$P$ & 3 & 76 & 17,2 & 5,172 & 1,724 & & & & & & & & & & 100 \\
\hline $\mathrm{F}$ & & & 1 & & & & & & & & & & & & 1 \\
\hline$P$ & 4 & & 100 & & & & & & & & & & & & 100 \\
\hline $\mathrm{F}$ & & 104 & 76 & 23 & 9 & 2 & 3 & 1 & 1 & & 1 & & & 1 & 221 \\
\hline$P$ & 5 & 47 & 34,4 & 10,41 & 4,072 & 0,9051 & 1,357 & 0,452 & 0,452 & & 0,452 & & & 0,452 & 100 \\
\hline $\mathrm{F}$ & & 72 & 50 & 22 & 15 & 3 & 4 & 3 & 1 & 1 & & 1 & & & 172 \\
\hline$P$ & 6 & 42 & 29,1 & 12,79 & 8,721 & 1,7442 & 2,326 & 1,744 & $0,581 \mathrm{c}$ & 0,581 & & 0,581 & & & 100 \\
\hline $\mathrm{F}$ & & 112 & 31 & 22 & 12 & 5 & 2 & 1 & & & & & & & 185 \\
\hline$P$ & 7 & 61 & 16,8 & 11,89 & 6,4862 & 2,7031 & 1,081 & 0,541 & & & & & & & 100 \\
\hline$F$ & & 72 & 49 & 23 & 9 & 6 & 2 & 1 & 2 & & & & & & 164 \\
\hline$P$ & 8 & 44 & 29,9 & 14,02 & 5,488 & 3,659 & 1,22 & 0,61 & 1,22 & & & & & & 100 \\
\hline $\mathrm{F}$ & & 83 & 52 & 18 & 12 & 5 & 3 & 1 & 2 & 1 & 1 & & 1 & & 179 \\
\hline P & 9 & 46 & 29,1 & 10,06 & 6,7042 & 2,7931 & 1,676 & 0,559 & 1,117 & 0,5590 & 0,559 & & 0,559 & & 100 \\
\hline$F$ & & 28 & 17 & 13 & 2 & 4 & 1 & 2 & & 1 & & & & & 68 \\
\hline$P$ & 10 & 41 & 25 & 19,12 & 2,941 & 5,8821 & 1,471 & 2,941 & & 1,471 & & & & & 100 \\
\hline$F$ & & 83 & 35 & 10 & 8 & 5 & 3 & 1 & 3 & & & & & & 148 \\
\hline$P$ & 11 & 56 & 23,6 & 6,757 & 5,405 & 3,3782 & 2,027 & 0,676 & 2,027 & & & & & & 100 \\
\hline $\mathrm{F}$ & & 102 & 34 & 18 & 8 & 5 & 1 & 1 & 1 & 1 & & & & & 171 \\
\hline$P$ & 12 & 60 & 19,9 & 10,53 & 4,6782 & 2,9240 & 0,585 & 0,585 & 0,585 & 0,585 & & & & & 100 \\
\hline
\end{tabular}

F: Frecuencia. P: Porcentaje.

(a-h) Medias con diferente letra dentro de una misma columna son significativamente diferentes $(P<0,05)$

C: café A: aserrín S: bagazo de caña de azúcar M: tallo de maíz. 
Tabla 7. Eficiencia biológica (\%) de P. ostreatus para 12 tratamientos (Cosechas 1, 2 y Total).

\begin{tabular}{|c|c|c|c|c|c|}
\hline \multirow[b]{2}{*}{ Tratamiento } & \multirow[b]{2}{*}{ Sustrato } & \multirow[b]{2}{*}{$\%$ en volumen } & \multicolumn{3}{|c|}{ Eficiencia biológica } \\
\hline & & & Cosecha 1 & Cosecha 2 & Total \\
\hline 1 & $\mathrm{C}$ & $(100)$ & $16,0^{a}(1,7)^{*} a$ & $11,7^{a}(1,0)^{*} a, d$ & $27,7^{a}(2,7)^{*} a, c$ \\
\hline 2 & A & $(100)$ & $4,8(5,6) b$ & $0,0(0,0) \mathrm{b}$ & $4,8(5,6) b$ \\
\hline 3 & $S$ & $(100)$ & $16,8(4,9) a, c$ & $4,0(2,8) b, c$ & $20,8(3,7) c$ \\
\hline 4 & M & $(100)$ & $0,5(1,1) b$ & $0,0(0,0) \mathrm{b}$ & $0,5(1,1) b$ \\
\hline 5 & $C+A$ & $(75+25)$ & $20,2(3,4) a, d$ & $13,4(1,9) a, d$ & $33,7(1,5) a, d$ \\
\hline 6 & $C+A$ & $(50+50)$ & $24,6(4,2) a, e$ & $11,2(1,9) a, d$ & $35,8(3,8) a, e$ \\
\hline 7 & $C+A$ & $(25+75)$ & $22,5(2,9) a, f$ & $9,5(1,1) c, d$ & $32,0(3,4) a, f$ \\
\hline 8 & $C+A+M+S$ & $(25+25+25+25)$ & $34,1(3,1) e$ & $14,4(3,9) a, d$ & $48,4(3,4) \mathrm{g}$ \\
\hline 9 & $C+M+S$ & $(50+25+25)$ & $28,0(5,0) d, e, f$ & $17,0(3,7) a$ & $45,0(4,8) e, g$ \\
\hline 10 & $A+M+S$ & $(50+25+25)$ & $16,3(3,0) a, g$ & $12,9(3,0) a, d$ & $29,1(5,5) a, c, h$ \\
\hline 11 & $C+A+M$ & $(25+25+50)$ & $25,5(4,3) c, d, e, f, g$ & $13,8(3,7) a, d$ & $39,3(7,3) d, e, f, g, h$ \\
\hline 12 & $C+A+S$ & $(25+25+50)$ & $26,8(4,2) d, e, f$ & $14,1(2,2) a, d$ & $40,8(4,6) d, e, f, g$ \\
\hline
\end{tabular}

a Medias. *(Desviación estándar).

(a-h) Medias con diferente letra dentro de una misma columna son significativamente diferentes $(P<0,05)$.

C: café A: aserrín S: bagazo de caña de azúcar M: tallo de maíz.

Tabla 8. Productividad (kg (100 kg día) - 1) de P. ostreatus para 12 tratamientos (total de 2 cosechas).

\begin{tabular}{cllrr}
\hline \multirow{2}{*}{ Tratamiento } & Sustrato & \multicolumn{2}{c}{ \% en volumen } & \multicolumn{2}{c}{$\begin{array}{c}\text { Productividad } \\
\left(\mathbf{k g}\left(100 \mathbf{~ k g ~ d i ́ a ~}^{-1}\right)\right.\end{array}$} \\
\hline 1 & $\mathrm{C}$ & $(100)$ & $0,398^{\mathrm{a}}$ & $(0,1)^{*} \mathrm{a}$ \\
3 & $\mathrm{~S}$ & $(100)$ & 0,324 & $(0,0) \mathrm{a}$ \\
\hline 5 & $\mathrm{C}+\mathrm{A}$ & $(75+25)$ & 0,494 & $(0,0) \mathrm{a}$ \\
6 & $\mathrm{C}+\mathrm{A}$ & $(50+50)$ & 0,483 & $(0,0) \mathrm{a}$ \\
7 & $\mathrm{C}+\mathrm{A}$ & $(25+75)$ & 0,429 & $(0,0) \mathrm{a}$ \\
8 & $\mathrm{C}+\mathrm{A}+\mathrm{M}+\mathrm{S}$ & $(25+25+25+25)$ & 0,905 & $(0,0) \mathrm{b}$ \\
9 & $\mathrm{C}+\mathrm{M}+\mathrm{S}$ & $(50+25+25)$ & 0,742 & $(0,1) \mathrm{b}$ \\
10 & $\mathrm{~A}+\mathrm{M}+\mathrm{S}$ & $(50+25+25)$ & 0,451 & $(0,1) \mathrm{a}$ \\
11 & $\mathrm{C}+\mathrm{A}+\mathrm{M}$ & $(25+25+50)$ & 0,715 & $(0,2) \mathrm{b}$ \\
12 & $\mathrm{C}+\mathrm{A}+\mathrm{S}$ & $(25+25+50)$ & 0,721 & $(0,0) \mathrm{b}$ \\
\hline
\end{tabular}

a Medias. *(Desviación estándar).

(a-b) Medias con diferente letra dentro de una misma columna son significativamente diferentes $(P<0,05)$

C: café A: aserrín S: bagazo de caña de azúcar M: tallo de maíz 
de maíz con 42 días después de la inoculación. Respecto de la cobertura del micelio en cada sustrato, se observó que en los sustratos tallo de maíz solo y aserrín solo (tratamientos 2 y 4) no hubo una colonización homogénea como en los demás tratamientos; estos presentaron muchas superficies del sustrato libres de micelio como si se hubiera dado un especie de inhibición del crecimiento micelial del hongo; pero cuando estos materiales se presentaron en mezcla con café y con bagazo de caña de azúcar, tuvieron una mejor colonización micelial.

Estudios previos muestran que para P. ostreatus cultivado en sustratos sólidos esta fase debe durar cerca de 14 días con temperatura constante de $28^{\circ} \mathrm{C}$ en la oscuridad (19). En el presente estudio para los tratamientos con más rápida fase de incubación (tratamientos 8, 9, 10, 11 y 12) el número de días fue de 26 , estas diferencias probablemente fueron debido a que la temperatura del lugar en donde se hizo la fase de incubación en este ensayo no fue constante, sino que osciló entre $7^{\circ} \mathrm{C}$ y $34^{\circ} \mathrm{C}$.

El crecimiento y el valor nutricional de los hongos del género Pleurotus spp., depende principalmente del tipo de sustrato y condiciones del cultivo (20). Las diferencias observadas entre los tratamientos conformados por mezclas, el bagazo de la caña de azúcar y el tallo de maíz podrían haber mejorado algunas características físicas del sustrato tales como la aireación y drenaje ya que sus partículas son de mucho mayor tamaño respecto de las del café y el aserrín, lo que probablemente mejoró la porosidad en el sustrato y evitó la compactación que se pudo haber presentado en los tratamientos que no los presentaban y de esta forma permitirle al micelio del hongo una mayor y más rápida colonización del sustrato.

Probablemente se mejoró la distribución de la semilla en el sustrato en el momento de la inoculación. Lo que podría estar de acuerdo con lo observado por Zhang y colaboradores (21) quienes reportaron que el tamańo de las partículas tiene un efecto en el intercambio de aire entre los espacios del sustrato dentro de la bolsa con el exterior. Ellos encontraron en P. sajor-caju que al reducir el tamańo de partícula del sustrato se pudo dar una compactación del sustrato en la bolsa, por lo que el intercambio de aire se dificultó especialmente cuando las bolsas estuvieron cerradas; favoreciendo aumentos en las concentraciones de $\mathrm{CO} 2$ presumiblemente producidas por el hongo y disminución del $\mathrm{O}_{2}$ dentro del sustrato lo que tendría un efecto inhibitorio en el desarrollo del hongo.

Zadrazil y Puniya encontraron que la degradación del sustrato por parte del hongo no sólo depende de la calidad del mismo sino también del tamaño de la partícula, pues al reducir su tamaño, también se disminuye la difusión de gases en el sustrato (22). Al final del ensayo se observó que en los sustratos sin bagazo de caña de azúcar o tallo de maíz (café y aserrín solo y las combinaciones de estos), en el centro de éstos no se encontraba colonizado por el micelio, simplemente el hongo colonizó unos centímetros de profundidad, mientras que los sustratos que sí los presentaron en sus mezclas, se encontraron totalmente colonizados (tratamientos 8, 9, 10, 11 y 12).

Según Okano y colaboradores y Salmones y colaboradores el hongo en su fase de crecimiento micelial (fase de incubación) consume preferiblemente carbohidratos solubles y hemicelulosa respecto de la celulosa y lignina $(13,23)$. Con base en esto, otra razón podría ser que en los sustratos con mezclas, el bagazo de caña de azúcar o el tallo de maíz permitieron o aumentaron la disponibilidad de carbohidratos solubles o compuestos más fácilmente asimilables por el hongo en su fase de crecimiento micelial.

\section{Aparición de primordios}

Los resultados de este parámetro se pueden observar en la Tabla 3 y en la Figura 3. Se puede apreciar que los tratamientos que más rápidamente formaron primordios en la cosecha 1 fueron las mezclas de café con o sin aserrín junto con el bagazo de la caña de azúcar y/o el tallo de maíz, con formación de primordios entre 31 a 34 días después de la inoculación (tratamientos 8, 9, 11 y 12), los cuales presentaron diferencias estadísticamente significativas $(\mathrm{P}<0,05)$ con los demás tratamientos, a excepción del tratamiento 3 (bagazo de cańa de azúcar solo).

El tratamiento 10 (aserrín con bagazo de caña de azúcar y tallo de maíz) el cual fue uno de los tratamientos que menos días gastó de incubación, demoró esta vez 15 días más respecto de los tratamientos $8,9,11$ y 12 . Se puede resaltar que de estos tratamientos, tres de ellos, -8,11,12, además de ser mezclas de todos los tipos de residuos usados, presentan el café en baja proporción, Tabla 1. Los tratamientos que más tardaron en formar los primordios 
fueron el 2 y el 7, con 87 y 50 días respectivamente, aunque en esta ocasión el tratamiento 2 fue el único que presentó diferencias estadísticamente significativas respecto de los demás tratamientos.

En la formación de los primordios para la cosecha 2 se puede apreciar que los tratamientos 8,11 y 12 fueron los que menos días gastaron, 47, 49 y 50 días respectivamente, coincidiendo con lo mostrado en la cosecha 1 , a excepción del tratamiento 9, el cual tardó 6 días más y presentó diferencias estadísticamente significativas con el tratamiento 8 . Los tratamientos 2 y 4 no tuvieron segunda cosecha. No hubo diferencias estadísticamente significativas entre los tratamientos con café solo y en mezcla con aserrín de establo (tratamientos 1, 5, 6 y 7).

El número de días para la aparición de primordios en los hongos del género Pleurotus spp., generalmente ha sido observada entre los 22 a 28 días después de la inoculación. Kalmis y Sargin encontraron que el número de días para la aparición de primordios de Pleurotus cornucopiae var. citrinopileatus y P. sajor-caju cultivados sobre paja de trigo (el principal sustrato usado en la producción de Pleurotus $s p p$.) bajo condiciones controladas, con temperatura de $20{ }^{\circ} \mathrm{C}$ y 12 horas de luz artificial (600 lux) fue de 22 y 28 días respectivamente para una cosecha (24).

Salmones y colaboradores encontraron que el número de días para la aparición de primordios de P. ostreatus cultivado sobre paja de trigo y pulpa de café bajo condiciones controladas, con temperatura de $24^{\circ} \mathrm{C}$, humedad relativa de $84 \%$ y 12 horas de luz natural fue de 25 días en ambos casos para una cosecha (23). Curvetto y su grupo encontraron que el número de días para la aparición de primordios de cinco diferentes cepas de $P$. ostreatus cultivado sobre testas de semilla de girasol y suplementadas con amonio y manganeso bajo condiciones controladas con temperatura de $21^{\circ} \mathrm{C}$, humedad relativa entre $80 \%$ y $90 \%$ y 12 horas de luz (1500 a 2000 lux), estuvo entre 24 y 28 días para la primer cosecha (20).

Leifa y colaboradores encontraron que el número de días para la aparición de primordios de P. ostreatus cultivado sobre borra de café (residuo industrial de la extracción de solubles del café tostado) al 60\% de peso seco en mezcla con hojas de café, fue de 23 días para una cosecha (25). En el presente ensayo con temperaturas entre $7^{\circ} \mathrm{C}$ y $31,5^{\circ} \mathrm{C}$, humedad relativa entre $33 \%$ y $100 \%$ y
12 horas de luz, para los tratamientos con más rápida aparición de primordios (tratamientos 8, 9, 11 y 12) el número de días para la primera cosecha fue entre 31 y 34 días y para la segunda cosecha fue entre 48 y 55 días.

A pesar de que no fueron condiciones controladas y que fueron cultivados en diferentes sustratos y diferentes cepas, el número de días para la aparición de primordios para la primera cosecha en estos tratamientos fueron cercanos a los encontrados por los investigadores anteriormente nombrados, inclusive con el número de días que se reportó para la primer cosecha en el sustrato de paja de trigo.

En cuanto a las diferencias entre tratamientos se podrían dar las mismas razones respecto de las características que pueden generar las mezclas de residuos en los sustratos. A excepción de que en este caso el tratamiento 10 (aserrín con bagazo de caña de azúcar y tallo de maíz) no fue uno de los mejores tratamientos, con lo anterior y con lo mostrado cuando se usó este residuo solo y en combinación con el café, se podría afirmar que el aserrín no tuvo un efecto benéfico en el número de días en incubación, ni en el número de días en la aparición de primordios. En los últimos días de la fase de fructificación (después de la segunda cosecha) se observó la presencia, aunque en muy baja cantidad, de mohos verdes y de gran diversidad de artrópodos en todos los tipos de residuos usados como sustratos, a excepción del residuo tallo de maíz solo (tratamiento 4) que únicamente presentó artrópodos; por lo que no se descartó ninguna unidad.

\section{Rendimiento}

Los resultados de este parámetro se pueden observar en la Tabla 4 y en la Figura 4. Se puede apreciar que los tratamientos que presentaron mayor rendimiento (g) en la cosecha 1 fueron en los que estuvo presente el café tanto solo como en mezcla (tratamientos 1, 5, 6, 7, 8, 9, 11 y 12) con una producción entre el 58\% y 70\% (suma de dos cosechas para cada tratamiento) y tuvieron diferencias estadísticamente significativas $(\mathrm{p}<0,05)$ respecto de los tratamientos que no tenían café (tratamientos 2, 3, 4 y 10).

El tratamiento 6 fue el que mayor rendimiento tuvo, con 262,8 g, pero no presentó diferencias estadísticamente significativas con los tratamientos $1,5,7,8$ y 9 . En cuanto a la cosecha 2, los tratamientos que tuvieron mayor rendimiento siguen siendo los que tenían café tanto solo 
como en mezcla (tratamientos $1,5,6,7,8,9,11$ y 12 ) con producciones entre el $30 \%$ y el $42 \%$, presentando diferencias estadísticamente significativas respecto de los demás tratamientos. En esta cosecha el tratamiento que tuvo mayor rendimiento fue el 5 con $163,3 \mathrm{~g}$ pero no presentó diferencias significativas con los tratamientos 1,6 y 9 .

También se pudo observar que en estos tratamientos (los que presentaban café) la disminución del rendimiento entre la cosecha 1 y 2 fue mayor para los tratamientos que presentaban el café en bajas proporciones en porcentaje de peso seco (tratamientos 6, 7, 8, 11 y 12), con bajas en la producción entre el $48 \%$ y $58 \%$ respecto de los tratamientos que presentaban el café en altas proporciones (tratamientos 1, 5 y 9), con bajas en la producción entre $27 \%$ y $39 \%$. Las proporciones en peso seco de los sustratos se dan en la Tabla 1.

Lo anterior podría sugerir que el principal ingrediente que aporta para la producción de los cuerpos fructíferos es el café, ya que tanto solo como en mezcla presentó los mayores rendimientos tanto para la cosecha 1 como para la cosecha 2. También se podría decir que el rendimiento bajó más de una cosecha a otra en forma inversa respecto de la cantidad de café presente en los sustratos. Los tratamientos en los que no estuvo presente el café (tratamientos 2, 3,4 y 10) fueron los que presentaron los más bajos rendimientos siendo el sustrato con solo tallo de maíz (tratamiento 4) el de más bajo rendimiento con $1,5 \mathrm{~g}$ para el total de dos cosechas, lo que sugeriría que este residuo no aportó nutrientes para el desarrollo de los cuerpos fructíferos, además de que este tratamiento no presentó un buen crecimiento micelial en su fase de incubación; esto probablemente a que los tallos de maíz estuvieron un tiempo considerable a la intemperie después de ser cosechados, también duraron aproximadamente 5 meses almacenados y se observó que este residuo permaneció con algo de humedad, lo que de alguna manera pudo haber afectado el desarrollo del hongo, este tipo de residuo fue el único en donde no se desarrollaron mohos verdes.

En un estudio realizado por Baysal y colaboradores (26), se evaluó un cultivo de $P$. ostreatus usando como sustrato diferentes mezclas de papel con otros materiales entre ellos la gallinaza; observaron que al adicionar gallinaza al papel, se prolongaban los días de incubación y de aparición de primordios y que los rendimientos disminuían, probablemente debido a los altos contenidos de nitrógeno de la gallinaza. Este pudo haber ocurrido con el aserrín, ya que este provenía de establos de animales y presentaba estiércol; y cuando se uso sólo como sustrato (tratamiento 2) fue el que más días tardó en el periodo de incubación y en la aparición de primordios. Aunque cuando se utilizó el aserrín mezclado con bagazo de caña de azúcar y con tallo de maíz (tratamiento 10), fue uno de los sustratos que menos demoró en cuanto al número de días en incubación, probablemente debido a la presencia del bagazo de la caña de azúcar, Tablas 2 y 3.

Los tratamientos de mayor rendimiento y con diferencias estadísticamente significativas, fueron los presentados en 1, 5, 6 y 9, con mayor cantidad de café en sus mezclas respecto de los tratamientos 8,11 y 12 , con la excepción de que el tratamiento 8 fue el único tratamiento con café en baja proporción que no presentó diferencias estadísticamente significativas con los tratamientos 1, 6, y 9. Los tratamientos 8, 9, 11 y 12 presentaron dos cosechas más (datos no procesados ni exhibidos).

\section{Frecuencia y porcentaje del peso de cada cuerpo fructífero}

Los pesos de cada cuerpo fructífero se clasificaron cada $5 \mathrm{~g}$, de acuerdo a como se muestra en la Tabla 5. Se muestran la frecuencia y porcentaje del peso de cada cuerpo fructífero para la suma de las dos cosechas en la Tabla 6. Se observa que en todos los tratamientos entre el 67\% al 98\% de los cuerpos fructíferos pesaron de $1 \mathrm{~g}$ a $15 \mathrm{~g}$, en este mismo rango el diámetro de los sombreros fue de $3 \mathrm{~cm}$ a $8 \mathrm{~cm}$ (datos no exhibidos).

\section{Eficiencia biológica}

Los resultados obtenidos se dan en la Tabla 7 y en la Figura 5. Se puede apreciar que los tratamientos con mayor eficiencia biológica (\%) para la cosecha 1 , fueron los tratamientos en los que estuvo el café en proporciones de bajas a medias, 25\% y $50 \%$ respectivamente (tratamientos $6,8,9,11,12$ ), encontrándose la mayor eficiencia en el tratamiento 8 con una eficiencia biológica del 34\% y siendo este el único con diferencia significativa $(\mathrm{p}<0,05)$ respecto de los demás tratamientos (tratamientos 1, 2, 3, $4,5,6,7$ y 10$)$. 
El tratamiento con menor eficiencia biológica de los que presentaban café fue el tratamiento 1 (solo café) con $16 \%$, siendo inclusive menor que el tratamiento 3 (solo bagazo de caña de azúcar) con $16.8 \%$. En cuanto a la eficiencia biológica de la cosecha 2 , se puede apreciar que en todos los tratamientos que contenían café no existieron diferencias significativas, tampoco hubo diferencias con el tratamiento 10 (mezcla de aserrín, tallo de maíz y bagazo de caña de azúcar) el cual no presentaba café. Se podría decir que si bien el café es el principal residuo que aporta para la producción de cuerpos fructíferos, no necesariamente a mayores cantidades se van a obtener mayores rendimientos en términos de eficiencia biológica.

En cuanto a la eficiencia biológica total, Tabla 7 y Figura 5, se puede decir que los mejores tratamientos fueron en los que estuvo mezclado el café en bajas proporciones (25\% y $50 \%$ ) con o sin aserrín y con cualquier residuo entre el tallo de maíz y el bagazo de la caña de azúcar (tratamientos 8, 9, 11 y 12), con eficiencias biológicas que van desde el $40 \%$ hasta el $48 \%$ para los tratamientos 11 y 8 respectivamente y los cuales presentaron diferencias significativas $(\mathrm{P}<0,05)$ con el tratamiento uno (solo café), con eficiencia biológica del $28 \%$.

La calidad productiva de un sustrato se percibe como aceptable a partir de eficiencias biológicas del 50\% (27). Job y su equipo reportó que las eficiencias biológicas de $P$. ostreatus cultivado sobre borra de café al 18 y $55 \%$ de peso seco en mezcla con aserrín y paja de trigo, también sobre trigo en mezcla con aserrín bajo condiciones controladas con temperatura de $17{ }^{\circ} \mathrm{C}$, humedad relativa de $95 \%$ y 8 horas luz (1100 lux), fueron de 40,5, 43,2 y 35,2\% respectivamente para dos cosechas, calificándolas como aptas para uso comercial (28).

Vega y colaboradores reportaron en un ensayo donde evaluaron diferentes cepas de Pleurotus djamor sobre sustratos de paja de arroz, eficiencias biológicas de 57,7\% y $61,3 \%$ calificándolas como aptas para uso comercial (29). Aunque las eficiencias biológicas en este ensayo fueron bajas, estuvieron cercanas a las aceptadas comercialmente por los investigadores anteriormente nombrados, en el caso de los mejores tratamientos (tratamientos 8, 9, 11 y 12).

\section{Productividad}

Los resultados se muestran en la Tabla 8. Estos se obtuvieron con base al peso acumulado de dos cosechas, la cantidad de sustrato seco y el número total de días para producirlos; se descartan los tratamientos 2 y 4 ya que de ellos sólo se obtuvo una cosecha. Se puede apreciar que los tratamientos más productivos y con diferencias estadísticamente significativas respecto de los demás, fueron las mezclas de café con o sin aserrín junto con el bagazo de la caña de azúcar y/o el tallo de maíz (tratamientos 8, 9, 11 y 12), siendo el tratamiento 8 el de mayor productividad con $0,905 \mathrm{~kg}$ de cuerpos fructíferos frescos producidos en $100 \mathrm{~kg}$ de sustrato seco por día; en los demás tratamientos $(1,3,5,6,7$ y 10) la productividad estuvo entre 0,324 y $0,494 \mathrm{~kg}$ de cuerpos fructíferos por $100 \mathrm{~kg}$ de sustrato seco por día.

Curvetto y colaboradores encontraron que la productividad de cinco diferentes cepas de $P$. ostreatus cultivado sobre testas de semilla de girasol y suplementadas con amonio y manganeso bajo condiciones controladas con temperatura de $21^{\circ} \mathrm{C}$, humedad relativa entre $80 \%$ y $90 \%$ y 12 horas de luz (1500 a 2000 lux), estuvo entre $1,2 \mathrm{~kg}$ y $3 \mathrm{~kg}$ de cuerpos fructíferos por $100 \mathrm{~kg}$ de sustrato seco por día (20). El tratamiento más viable en el presente ensayo fue el tratamiento 8 (café con aserrín, bagazo de caña de azúcar y tallo de maíz). Además, en este sustrato se utilizaron los dos residuos de difícil degradación en la zona de compostaje del campus universitario (aserrín de establos y café).

De acuerdo a los resultados obtenidos en las variables de rendimiento de un cultivo de $P$. ostreatus en el presente ensayo, se puede afirmar que los residuos lignocelulósicos evaluados, de difícil degradación en compostaje, como el residuo de café de consumo humano y el aserrín de establos de animales sirven como sustrato. El cultivo de $P$. ostreatus sobre estos residuos puede ser una alternativa para el manejo de los mismos, cuando estos se combinaron con residuos de partículas más grandes como lo fueron el bagazo de cańa de azúcar y/o el tallo de maíz. NO 


\section{Referencias}

1. Chang ST. World production of cultivated edible and medicinal mushrooms in 1997 with emphasis on Lentinus edodes (Berk). Sing in China. Inter J Med Mush. 1997;1:291-300.

2. Cardona, L.F. Anotaciones acerca de la bromatología y el cultivo del hongo comestible Pleurotus ostreatus. Crónica forestal y del medio ambiente. 2001;16:99-119.

3. Rodriguez N, Jaramillo C. Cultivo de hongos comestibles del género Pleurotus sobre residuos agrícolas de la zona cafetera" CENICAFÉ, Chinchiná, Colombia. Boletín técnico N²7. 2005.

4. Zadrazil, F. The ecology and industrial production of Pleurotus ostreatus, P. florida, P. cornucopiae and P. eryngii. Mush Sc 1974;9:621-652.

5. Sánchez JE, Royse DJ. La biología y el cultivo de Pleurotus spp. Ed. El colegio de la frontera sur y LIMUSA, México. 2001.

6. Rajarathnam S, Bano Z, Patwardhan M. Nutrition of the mushroom Pleurotus flabellatus during its growth on paddy straw substrate. J-Hortic-Sci. 1986;61:223-232.

7. Bermúdez R, Donoso C, Martínez CE, Ramos E, Morris H. Efecto de la luz en la concentración de micosteroles de Pleurotus ostreatus Var. Florida. Rev Cuba Aliment Nutr. 2002;16:13-18.

8. Miles PG, Chang ST. Biología de las setas: Fundamentos básicos y acontecimientos actuales, Bogotá. World Scientific. 1995. pg. 206.

9. Bautista M, M.G. Alanís, E. González, C.L. García. Composición química de tres cepas mexicanas de setas (Pleurotus ostreatus). ALAN. 1998;48:359-363.

10. Crisan E, Sands A. Nutritional Value. In: Chang ST, Hayes WA (Eds.) The Biology and cultivation of edible mushrooms. London, Academic Press.1978. pg.137-168.

11. Del Toro G, Castelán R, Garín-Aguilar ME, Leal H. Biological quality of proteins from three strains of Pleurotus spp. Food Chemistry. 2006;94:494-497.

12. Karunanandaa K, Varga GA, Akin DE, Rigsby LL, Royse DJ. Botanical fractions of rice straw colonized by white-rot fungi: changes in chemical composition and structure. Animal Feed Science and Technology. 1995;55:179-199.

13. Okano K, Fukui S, Kitao R, Usagawa T. Effects of culture length of Pleurotus eryngii grown on sugarcane bagasse on in vitro digestibility and chemical composition. Anim. Feed Sci Technol, 2007;136:240-247.

14. Serna YB, Hinestroza LI, Ríos A, Escobar R, Hinestroza L, Arango R, Mena VE, Aguilar Y, Bonilla JA. Producción de limoncillo, Cymbopogon citratus en sustratos resultantes de la producción de la seta (Pleurotus sajo-cajú) en Quibdó. Rev Institu Universidad Tecnológica del Choc 2005;22:63-68.

15. Masaphy S, Levanon D, Henis Y. Degradation of atrazine by the lignocellulolytic Pleurotus pulmonarius during solid-state fermentation. Bioresource Technology 1996;56:207-214.
16. Sarangi I, Ghosh D, Bhutia SK, Mallick SK, Maiti TK. Anti-tumor and immunomodulating effects of Pleurotus ostreatus mycelia-derived proteoglycans. Int Immunopharmacol. 2006;6:1287-1297.

17. Delfín-Alcalá I, Durán-De-Bazúa C. Biodegradación de residuos urbanos lignocelulósicos por pleurotus. Rev Int Contam Ambient. 2003; 19:37-45.

18. Bonilla CI, González WM. Caracterización y evaluación de los residuos sólidos biodegradables producidos en el campus universitario como sustrato en el proceso de compostaje. Tesis de grado para optar por el título de Ingeniería Agronómica. Facultad de Agronomía. Universidad Nacional de Colombia. 2004.

19. Soto-Cruz O, Saucedo-Castañeda G, Pablos-Hach JL, GutiérrezRojas M, Favela E. Effect of substrate composition on the mycelial growth of Pleurotus ostreatus. An analysis by mixture and response surface methodologies. Process Biochemistry 1999;35:127-133.

20. Curvetto NR, Figlas D, Devalis R, Delmastro S. Growth and productivity of different Pleurotus ostreatus strains on sunflower seed hulls supplemented with N-NH4+ and/or Mn(II). Bioresour Technol. 2002;84:171-176.

21. Ruihong Z, Xiujin L, Fadel JG. Oyster mushroom cultivation with rice and wheat straw. Bioresource Technology. 2002;82:277-284.

22. Zadrazil F, Puniya AK. Studies on the effect of particle size on solid-state fermentation of sugarcane bagasse into animal feed using white-rot fungi. Bioresource Technology. 1995;54:85-87.

23. Salmones D, Mata G, Waliszewski KN. Comparative culturing of Pleurotus spp. on coffee pulp and wheat straw: biomass production and substrate biodegradation. Bioresour Technol. 2005;96:537-544.

24. Kalmis E, Sargin S. Cultivation of two Pleurotus species on wheat straw substrates containing olive mill waste water. Int Biodeterior Biodegradation. 2004;53:43-47.

25. Leifa F, Pandey A, Soccol CR. Solid state cultivation--an efficient method to use toxic agro-industrial residues. J Basic Microbiol. 2000;40:187-197.

26. Baysal E, Peker H, Yalinkiliç MK, Temiz A. Cultivation of oyster mushroom on waste paper with some added supplementary materials. Bioresour Technol. 2003;89:95-97.

27. Sánchez JE, Orozco GM, Hernández D, Nieto MG, Márquez FJ. Capacidad del género Pleurotus para la degradación del insecticida endosulfán. El Cromosoma. Boletín del Colegio de Biotecnólogos de Chiapas. 2006;2:31-120.

28. Job D. La utilización de la borra del café como substrato de base para el cultivo de Pleurotus ostreatus (Jacq.:Fr.) Kummer. Rev Iberoam Micol 2004;21:195-197.

29. Vega, A., G. Mata, D. Salmones y R.E. Caballero. Cultivo de cepas nativas de Pleurotus djamor en Panamá, en paja de arroz y pulpa de café. Revista Mexicana de Micología. 2006;23:93-9. 